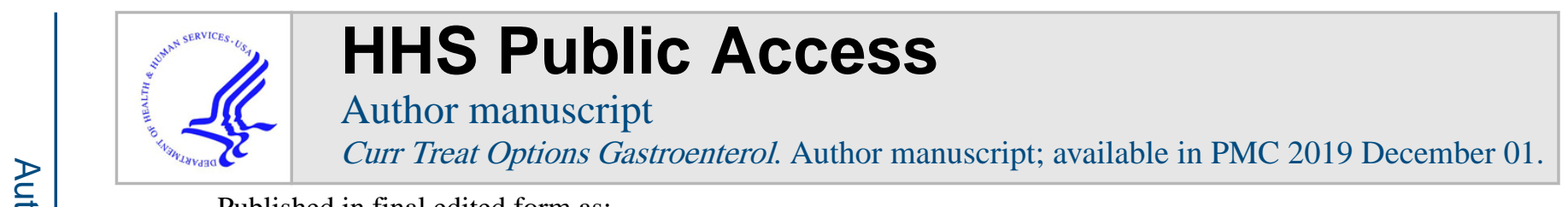

Published in final edited form as:

Curr Treat Options Gastroenterol. 2018 December ; 16(4): 466-478. doi:10.1007/s11938-018-0197-8.

\title{
Pancreatic Cancer and Diabetes Mellitus
}

\author{
Ayush Sharma, MBBS and Suresh T. Chari, MD \\ Division of Gastroenterology and Hepatology, Mayo Clinic, Rochester, MN, USA
}

\begin{abstract}
Purpose of review: The relationship between pancreatic ductal adenocarcinoma (PDAC) and diabetes mellitus (DM) is complex. We reviewed the recent medical literature regarding the effect of anti-diabetic medication on PDAC risk and survival; risk of PDAC in DM; and role of DM in early detection of PDAC.
\end{abstract}

Recent findings: Studies report that while some anti-diabetic medications (e.g., metformin) may decrease the risk of PDAC, others (insulin, sulfonylureas and incretin-based therapies) may increase the risk. However, these observations may be subject to protopathic biases. Metformin's anti-tumor activity may have influence overall survival of PDAC, but epidemiological reports have largely been inconsistent to defend these findings due to heterogeneous methodologies. There is congruent data to support the association between DM and PDAC, with an inverse relationship to DM duration. Older subjects with new-onset DM are the only known high-risk group for PDAC and strategy using this group for early detection has led to development of clinical risk prediction models that defines a very high-risk PDAC group.

Summary: Role of anti-diabetic medication in PDAC risk modification or survival is controversial. With successful efforts to distinguish type 2-DM from PDAC-DM using risk stratifying models, there is an opportunity to initiate screening protocols for early detection of PDAC in a sub-set of DM subjects.

\section{Keywords}

Pancreatic cancer; new-onset diabetes; anti-diabetic medication; early detection; hyperglycemia

\section{Introduction}

The relationship between diabetes mellitus (DM) and pancreatic ductal adenocarcinoma (PDAC) has been known for more than a century. However, understanding of this relationship is complicated with existence of bidirectional link between the two conditions. Long-standing DM (LSDM) has been well established as a risk factor for development of PDAC, but growing epidemiologic, clinical and experimental evidence suggest that new-

\footnotetext{
Corresponding author: Suresh T. Chari, M.D., Division of Gastroenterology and Hepatology, Mayo Clinic College of Medicine and Sciences, 200 First St SW, Rochester, MN 55905, Phone: 507-255-5713, Fax: 507-255-6318, chari.suresh@ mayo.edu.

Conflict of Interest

Suresh Chari reports grants from NIH and the Kenner Family Research foundation, during the conduct of the study. Ayush Sharma declares no conflict of interest.

Human and Animal Rights and Informed Consent

This article does not contain any studies with human or animal subjects performed by any of the authors.
} 
onset DM (NOD) is a paraneoplastic phenomenon in a subset of patients that precedes PDAC diagnosis [1]. Recognizing NOD as a clinical manifestation of occult PDAC has important implication in the field of early detection of PDAC. Ability to distinguishing NOD caused by PDAC, which is distinct from NOD of type 2 provides an opportunity to identify and diagnose PDAC at a stage at which curative therapy is possible. More recent, studies also suggest anti-diabetic medications (ADM) directly affect the key factors mediating the association between type 2-DM and PDAC, with an influence on cancer development, progression and outcome [20]. This has led to increasing interest in understanding the causal relationship between ADM use and the risk of PDAC and whether it has a role in PDAC prevention and treatment. Here, we discuss the role of ADMs in the risk modification of PDAC and its influence on overall survival of PDAC along with a current opinion in the field of DM and PDAC. We also summarize the advances made in the field of early detection of PDAC in the past year and selected the best publications, which merited attention, for this review.

\section{PDAC risk and anti-diabetic medications}

Epidemiological studies have found that ADM agents reducing insulin resistance (such as metformin and thiazolidinedione) reduce risk of PDAC, whereas ADM agents increasing levels of circulating insulin (such as sulfonylureas [insulin secretagogues] and insulin analogs) are associated with an increased risk for PDAC[3-6]. Data on relatively new ADMs, incretin-based analogues, is sparse, with conflicting findings and no clear association [7-9]. Although, results of current epidemiological studies suggest a relationship between ADM use and PDAC risk, these data should be inferred with caution.

\section{Metformin use and risk of PDAC}

Metformin is the most widely used ADM for type 2 DM. Metformin acts by decreasing circulating insulin and insulin-like growth factors (IGF) levels and causes inhibition of mitochondrial glycerophosphate dehydrogenase and increases AMP-activated protein kinase (AMPK), consequently leading to an altered hepatocellular redox state, reduced conversion of lactate and glycerol to glucose, and decreased hepatic gluconeogenesis [20, 10]. Chen et al showed that metformin suppressed cancer initiation and progression in genetic mouse model of PDAC [11]. Considering this, there is growing interest to investigate the role of metformin as a promising preventive agent for PDAC. Consistent with reports by Wang et al, the most recent meta-analysis reported on pooled data from published studies also found that risk of PDAC was lower in metformin users (Relative risk $[R R]=0.61$ ) $[12,13]$. However, the studies included in meta-analyses vary considerably in their methodology and don't account for the fact that PDAC can cause and/or worsen DM. Olson et al showed that there is worsening of DM months prior to PDAC diagnosis leading to initiation of insulin and therefore, altering the natural course of DM treatment in PDAC subjects [14]. In a large case-control observational study, Bodmer et al addressed this limitation by adjusting for two-year cancer latency period, and found no association between metformin use and future PDAC risk [5]. Similar observations were also noted in the two randomized clinical trials comparing metformin use with other ADMs or placebo for type 2 DM subjects [13]. Due to escalation of ADM therapy from metformin to more potent anti-glycemic agents in DM 
subjects with undiagnosed PDAC introduces unintended biases and leads to overestimation of metformin's protective effect. So far current epidemiological studies have been inconsistent in firmly establishing a protective effect of metformin therapy in PDAC.

\section{Insulin use and risk of PDAC}

Insulin therapy is often required with natural progression of type $2 \mathrm{DM}$. Insulin-glargine, the long acting insulin analog, is the most widely used long acting insulin. Experimental data suggests insulin has potential mitogenic and anti-apoptotic effects in cultured cancer cells through activation of the IGF-1 pathway $[15,16]$. A systematic review by Colmers et al, found that new use of insulin was associated with an increased risk of PDAC (RR, 3.18) [4]. A similar risk of PDAC is also reported with use of sulfonylureas (insulin secretagogues) routinely used in DM treatment $[13,5,6]$. Recently, these observations seem to be confirmed by Lee et al, who analyzed a Korean population-based cohort of subjects with and without DM and reported that risk of PDAC increases in patients after initiating therapy with sulfonylureas (HR, 1.73) and insulin (HR, 2.86) compared to no drug exposure group [17]. Some have speculated a direct causal relationship between insulin and PDAC occurrence secondary to anabolic effect of insulin on tumor cells [18]. However, if this were to be true, then a steadily increasing risk of PDAC should be noted with an increasingly longer exposure to these therapies. In contrast, all these studies show that increased risk is observed primarily in the months immediately after initiating therapy, suggesting an underlying undiagnosed PDAC causing uncontrolled DM. This is referred as protopathic bias, which arises when the initiation of a drug (exposure) occurs in response to a clinical manifestation of the disease (at this point undiagnosed) under study outcome [19]. Therefore, we believe, escalation of therapy to insulin for uncontrolled DM may be a sign of undiagnosed PDAC, especially when occurring in face of unintentional weight loss and worsening hyperglycemia [1].

\section{Incretin-based therapy and risk of PDAC}

Over the last decade, the worldwide prevalence of type 2 DM has increased the use of incretin-based drugs. There are two types of incretin-based drugs, glucagon-like peptide-1 receptor agonist (GLP-1) and dipeptidyl peptidase-IV (DPP-IV) inhibitors, both with pharmacological effects on the interaction between the gut and endocrine system [20]. While incretin-based therapy has a superior efficacy in controlling blood glucose compared to other ADMs in type $2 \mathrm{DM}$, studies suggest that these incretin mimetics may increase the risk of PDAC development [21-23]. There are various observational and randomized controlled clinical trials conducted to establish this association [22-26]. A meta-analysis by Chen et al reported from pooled data of randomized controlled trials showed incretin-based therapies is not associated with increased risk of PDAC (RR, 0.70) [7]. Interestingly, they report a lower incidence of pancreatic neoplasms in incretin-based groups than those in placebo or nonincretin-based ADMs in studies with longer follow-up period. In contrast, Boniol et al analyzed results from two large retrospective cohorts and observed that recent prescription of incretin therapy is associated with an increased risk of PDAC compared to other ADMs [8]. We believe the reason for an increase risk after incretin-based therapy is likely 
consequence of an occult PDAC that provokes or aggravates DM and therefore, confounds the risk association.

\section{PDAC survival and anti-DM medication}

There are numerous studies showing influence on survival with ADM use, but till date, effect of metformin or other ADMs on clinical outcomes in PDAC-DM patients remain controversial. Overall, the influence of ADM on PDAC survival is confounded by observation that DM itself is associated with poor outcomes in PDAC. Hart et al compared PDAC subjects with and without DM and reported that PDAC tumors with DM have a larger tumor size and higher CA19-9 levels, factors inversely related to PDAC prognosis [27]. In addition, Sharma et al showed that poorly differentiated PDAC tumors are more likely to be associated with worsening hyperglycemia and DM, which is well known to be associated with poorer outcomes [280].

\section{Effect of metformin on PDAC survival}

There are growing numbers of articles focusing on the effectiveness of metformin on survival of PDAC patients with DM. In last year, there are 4 meta-analyses and 2 observational studies addressing this relationship [29-34]. The four meta-analyses include a total of 15 unique studies (13 observational and 2 RCTs); and conclude that metformin in comparison to nonmetformin users in PDAC is associated with relative benefit in overall survival in observational studies, but not in randomized controlled studies [29-32]. In contrast, Frouws et al, who analyzed 907 patients with PDAC-DM, including 77 patients treated with metformin reported no survival benefit in patients with metformin use compared to non-users [33]. However, this study was limited by small proportion of patients with treatment on ADMs.

It is important to note that even though the results from meta-analyses support the notion that metformin use may improve the overall survival of PDAC-DM patients, most studies included are subject to immortal time bias. Immortal time is a span of cohort follow-up during which, because of exposure definition, the outcome under study could not occur. To control for this bias, Chaiteerakij et al used time-varying covariate analysis methods and showed no benefit of metformin therapy on PDAC overall survival [3500]. When the analysis was performed by using the conventional Cox model, an artificial survival benefit of metformin was detected [3500]. This highlights the importance of patient selection and appropriate statistical analysis methods when studying medication exposure and cancer survival.

\section{Effect of other ADMs on PDAC survival}

While there are several studies looking at survival benefit with metformin in PDAC subjects, there are few reports related to other oral hypoglycemic agents and insulin use. Zhou et al investigated the association between ADMs (insulin, sulfonylurea and thiazolidinedione) treatment and survival of PDAC and found no benefit of insulin, sulfonylureas or thiazolidinedione [30]. So far, there is no data supporting any survival benefit with incretinbased therapy. 


\section{PDAC risk and diabetes}

About $45 \%$ to $65 \%$ of patients with PDAC have DM at diagnosis, and the proportion of patients presenting with new-onset DM is nearly $25 \%$. Conversely, there is consistent data to support that DM imparts a two-fold increase risk for PDAC. However, this risk is influenced by DM duration and method of ascertainment of DM used to estimate PDAC risk.

\section{Risk of PDAC changes with duration of DM}

It is now well established, based on several observational studies and meta-analyses, that individuals with DM have an increased risk for PDAC compared to non-DM subjects, with risk being inversely associated with DM duration. The latest meta-analysis by Tan et al systematically reviewed and included all studies in last 10 years $(n=11 ; n=14,399)$ and concluded that DM was associated with a two-fold increased risk for PDAC (RR, 1.63) [36]. When stratified based on DM duration, they showed that patients with recent onset DM $(<2$ years) had higher incidence of PDAC compared to patients with 2-5 years of DM. These findings were confirmed by Makhoul et al who reported similar risk association in the Veterans Administrative (VA) database after controlling for matching factors (age, gender, body mass index etc.) between DM and no DM subjects (HR, 2.17) [37]. As expected, they also noted the inverse relationship of PDAC risk with DM duration, with increased incidence of PDAC persisting only in the first 5-years after DM diagnosis. This highlights the importance of distinguishing new-onset DM (NOD, defined as DM of $<3$ years duration) from long-standing DM (LSDM defined as DM of $>3$ years duration) when estimating PDAC risk.

Clinical and experimental data suggest that NOD is a cancer effect (paraneoplastic clinical manifestation), whereas LSDM is modest pathogenic risk factor [1]. Pannala et al showed amelioration of NOD, but not LSDM, following resection of PDAC, which further supports this observation [38]. Therefore, it is not surprising that compared to general population the NOD population has a 6-8-fold higher 3-year risk (high risk group), whereas LSDM has 1.5-2.0-fold lifetime risk (low risk group) (Figure 1) [1, 39, 40]. Even though, all these observational cohort studies and meta-analyses clearly establish the risk association between DM and PDAC, by contaminating LSDM with NOD, they seriously underestimate the overall PDAC risk.

\section{Degree of Risk of PDAC is dependent on method of ascertainment of DM}

A proportion of patients with pre-DM progresses to DM over time. Physicians, knowing that pre-DM and DM may be reversible or delayed by weight loss and exercise, tend not to label patients as having DM until lifestyle changes fail to work. Thus, physician diagnosis of NOD may be delayed by months to years after patients meet glycemic criteria for new-onset DM, which is often euphemistically coded as "hyperglycemia", "pre-diabetes" or "glucose intolerance". This has significant implications for its utility for prediction of early PDAC.

Aggarwal et al showed that in a primary care setting, in comparison to type 2 NOD, a disproportionately higher proportion of PDAC subjects with NOD fail to receive a DM diagnosis, due to the relatively short duration of DM before PDAC [4100]. This 
erroneously reduces the risk of PDAC in NOD. The 3-year cumulative incidence of PDAC in studies by Munigala et al, Boursi et al, Mokhoul et al and Pang et al, all using physician diagnosis of NOD have consistently observe a risk of $0.25 \%-0.40 \%$, significantly lower (by nearly half) than that reported in studies using glycemic criteria for NOD by Chari et al and Sharma et al (0.85\%-1.25\%) [42, 4300, 37, 44, 39, 4500]. This highlights that using physician diagnosis-NOD underestimates the incidence of PDAC in DM. Aggarwal et al also reported that physician diagnosis-NOD is closer to PDAC diagnosis than the glycemic onset of NOD with median onset from onset to diagnoses being 3 months (range, 1 to 14 months), thus shortening the already narrow "window of opportunity" to intercept PC early [410)].

Onset of DM may occur 4 to 7 years before its clinical diagnosis [46]. Fraser et al reported a delay of 3.7 years between initial hyperglycemia and clinician's diagnosis date of DM and delay in diagnosis was attributed to clinician inertia (provider factors) rather than patient factors [47]. Thus, new physician diagnosis of DM should not be confused with new-onset DM. As alluded earlier, there is a 1.5-fold increase risk of PDAC in LSDM patients compared to 6 to 8 -fold risk in NOD patients, and a delay in physician diagnosis of DM by $\sim 3$ years, likely reflects a PDAC risk in LSDM than true NOD. This would again lead to falsely low estimation of PDAC risk in NOD. For a successful early detection strategy using NOD as the trigger, it is important to use glycemic-defined rather physician diagnosed NOD and we advocate the use of definition of the American Diabetes Association for DM.

\section{Risk of PDAC in pre-DM}

Pre-DM is defined by fasting plasma glucose levels of $100-125 \mathrm{mg} / \mathrm{dL}$, a 2-h plasma glucose level of 140-100 mg/dL, or a glycosylated $\mathrm{Hb}$ of 5.7-6.4\% [48]. Recently Sharma et al investigated the pre-clinical progression of invasive PDAC in a case-control design using blood glucose levels as a biomarker for PDAC [280]. With 6-montly fasting blood glucose level data up to 60 months prior to PDAC diagnosis in cases and age- and gender-matched controls, they showed that PDAC-induced hyperglycemia develops $\sim 36$ months prior to cancer diagnosis. From an early detection standpoint, these observations suggest that distinguishing PDAC-induced hyperglycemia from pre-DM of type 2 could lead to earlier detection of PDAC. However, this will require a highly specific biomarker/clinical model to enrich the pre-DM cohort for PDAC, as nearly 86 million adults in the US are believed to have pre-DM [48]. Optimistically, with recent developments in identifying very-high risk groups for PDAC within NOD, similar strategies may be applied to subjects with pre-DM to help detect early PDAC.

\section{Role of DM in early detection of PDAC}

PDAC has a dismal (9\%) 5-year survival, largely because the majority (85\%) of PDAC is diagnosed at an advanced stage. Developing strategies for early detection of resectable sporadic PDAC are critical for improving survival. Since PDAC is uncommon, a 3-step sieve approach $(\boldsymbol{D E F})$ to its early detection has been suggested: (1) Define a high-risk group for PDAC; (2) Enrich the high-risk group further for PDAC and (3) Find the lesion in the highly enriched cohort [49]. 


\section{Define: new-onset diabetes as the first sieve}

Screening for sporadic PDAC has so far been considered unrealistic because of its low incidence in the general population, and lack of well-defined high-risk groups to screen for sporadic PDAC. The only known high-risk group for sporadic PDAC is that of subjects $>50$ years of age with NOD [39]. Chari et al first reported in a population-based study that approximately $1 \%$ of NOD (defined by glycemic criteria) will be diagnosed with PDAC in 3-year period [39]. Sharma et al recently validated these findings in another populationbased study using similar glycemic criteria for NOD [4500]. These observations suggest that DM is a biomarker of early-stage PDAC and identifying patients with NOD at their onset would offer the opportunity of early detection. However, NOD is relatively common with an expected annual incidence in US being 1\% [50]. The population of US over the age of 50 years is 108 million; of which $1 \%$ will have incident NOD (i.e., $~ 1.1$ million) [51]. Based on estimates by Chari et al and Sharma et al, $1 \%$ of this NOD population will be diagnosed with PDAC i.e. approximately 11,000 subjects. Conversely, expected rate of sporadic PDAC in US is 55,400 and NOD forms $\sim 20 \%$ of all PDAC, which comprises of 11,100 subjects. This puts into perspective that screening in NOD subjects for PDAC would potentially capture one-fourth of sporadic PDAC.

\section{Enrich: clinical models and biomarkers}

Since type $2 \mathrm{DM}$ is a very prevalent disease, screening for PDAC in this population may not be feasible. Therefore, it is important to enrich the NOD population further to identify individuals who would benefit most from screening. Boursi et al developed a risk prediction model for PDAC in NOD population using The Health Improvement Network (THIN) database UK [4300]. In their cohort of 109,385 NOD subjects, $0.4 \%$ was diagnosed with PDAC within 3 years of NOD diagnosis. Their final prediction model was based on demographic, behavioral, and clinical variables with predicted risk threshold of $44.7 \%$ sensitivity, $94 \%$ specificity, and a positive predictive value of $2.6 \%$ (at $1 \%$ over 3 years). However, the model used one physician diagnosis of NOD, which may have underestimated their baseline disease prevalence to $\sim 0.4 \%$ as explained earlier. Sharma et al, developed a clinical model called Enriching New-onset Diabetes for Pancreatic Cancer (ENDPAC) score using glycemic definition of NOD [4500]. The model used 3 parameters; age, change in blood glucose and delta weight loss and risk stratified the NOD subjects into 3 groups based on 3-year PDAC risk: low $(<0.1 \%)$, intermediate $(\sim 0.5 \%)$ and very high $(\sim 4 \%)$. The score of $>3$ had a sensitivity and specificity of $80 \%$. However, the study was limited by small numbers of PDAC events in the validation cohort and needs confirmation in other large cohorts before being implied in clinical practice. The development of these clinical models shows encouraging preliminary results in differentiating T2-NOD from PDAC-NOD.

The only available biomarker that is currently used clinically in PDAC is CA19-9. Choe et al evaluated the utility of CA19-9 levels as a screening indicator of PDAC in asymptomatic NOD subjects ( $\mathrm{n}=5111$ asymptomatic NOD of which 87 [1.7\%] developed PDAC) and reported in patients, especially with high serum bilirubin, CA19-9 was a useful marker for predicting cancer [52]. Study by Murakami et al, proposed a cut-off value for CA19-9 for PDAC screening in DM subjects using a matched case-control design and showed serum 
CA19-9 levels of $>75 \mathrm{U} / \mathrm{mL}$ had a sensitivity and specificity of $69.5 \%$ and $98.2 \%$, respectively to classify DM patients with or without PDAC [53]. However, it is well known that the usefulness of CA19-9 fades with increasing lead time and its power to discriminate between two types of DM will likely be low during the pre-diagnostic phase of PDAC [54]. Balasenthil et al, investigated genomic-based plasma TFP1/TNC-FN III-C/CA19-9 panel and noted it improved CA19-9 performance in all early stage cohorts, including discriminating stage IA/IB/IIA, stage IIB and all early stage cancers from healthy controls [55]. They concluded TFP1/TNC-FN III-C migration signature adds statistical significance to CA19-9's predictive power to detect early stage PDAC. But, it is important to note that these biomarkers are tested in samples collected at PDAC diagnosis which may not reflect metabolic abnormalities occurring during the pre-diagnostic phase. Therefore, whether these biomarkers maintain their predictiveness with increasing lead time needs to be validated in pre-diagnostic samples.

\section{Find: imaging in very-high risk cohort}

Recently Sharma et al determined if a tumor volume threshold was associated with PDAC induced-hyperglycemia and noted that hyperglycemic signal is strongest with larger tumors and fades with decreasing tumor volume [280]. The volume of smallest tumor associated with hyperglycemia was estimated to be 1.1 to $2.0 \mathrm{~mL}$ and this volume doubles 4 to 5 times in size by the point of cancer at diagnosis (average size: $8.0-12.0 \mathrm{~mL}$ ), providing ample of window of opportunity to find the lesion on imaging. Whether current imaging modalities (computerized tomography [CT], magnetic resonance imaging [MRI] or endoscopic ultrasound [EUS]) will be able to detect such tumors is yet unanswered. An important finding of that study was the largest diameter of PDAC tumors at the DM diagnosis was estimated to be $2-4 \mathrm{~cm}$, which provides hope that tumors at DM onset may be detected using CT or EUS. Results from prior studies by Pelaez-Luna et al and Gangi et al investigating the pre-diagnostic imaging scans suggest that nearly half of the PDAC subjects with CT scans obtained between 2 and 18 months prior to cancer diagnosis were identified as being diagnostic or suspicious for PDAC [56, 57]. These studies included scans done using low quality $\mathrm{CT}$ techniques and therefore may underestimate the actual findings. In addition, Pelaez-Luna et al results suggest that resectability of PDAC can be significantly improved if it is detected at least 6 months prior to its clinical diagnosis and that DM occurred at a resectable stage of disease [56]. This implies finding PDAC early in the NOD subjects should lead to a stage shift. As currently, $80 \%$ of PDAC at diagnosis have metastatic disease, and are unresectable, finding PDAC even 3 months before its diagnosis should lead to survival benefit beyond lead time.

\section{Feasibility of screening in NOD for PDAC}

Some may debate screening for PDAC in patients with NOD may not be feasible with risk being only $1 \%$; and imaging scanning of the pancreas as screening procedures would be neither cost-effective nor sensitive or specific [42]. To this end, Bruenderman et al estimated the screening cost for PDAC using MRI or magnetic resonance cholangiopancreatography for 3 years among individuals with NOD older than 50 years with weight loss and reported cost per added year ranges from $\$ 356.42$ based on Medicare costs to $\$ 1418.92$ based on 
national average [58 ]. In comparison to other solid organs (lung, breast, colon), screening for PDAC in this subset of NOD was reported to be affordable and cost-effective [59-61]. Moreover, with new developments in risk prediction models such as ENDPAC or UK THIN database, the targeted NOD population needing imaging significantly decreases the burden of screening.

\section{Conclusions}

Despite several reports, the role of ADM in risk association and influence on survival of PDAC remains controversial. The relationship of DM with PDAC is bi-directional with longstanding DM being a modest risk factor for development of PDAC whereas new-onset DM being an effect. Following principles of Define, Enrich and Find strategy for early detection of PDAC, there has been significant progress in the field, with identification of NOD as a high-risk population (Define), clinical risk prediction models distinguishing type 2-NOD from PDACNOD (Enrich) and opportunities to design future prospective protocols for interventional imaging studies in this enriched NOD cohort (Find). The hope is to capture a large percentage of those with resectable, early stage PDAC that are currently being diagnosed late.

\section{Grant support}

This work was supported by the NIH U01 Consortium for Study of Chronic Pancreatitis, Diabetes and Pancreatic cancer (CPDPC) (Chari) and Kenner Family Research Fund (Chari). The content is solely the responsibility of the authors and does not necessarily represent the official views of the National Institutes of Health or any other funding source.

\section{Abbreviations:}

ADM

DM

NOD

PDAC anti-diabetes medication

diabetes mellitus

new onset diabetes

pancreatic ductal adenocarcinoma

\section{References}

Papers of particular interest, published recently, have been highlighted as: ‘

- Of importance

O० Of major importance

1. Sah RP, Nagpal SJS, Mukhopadhyay D, Chari ST. New insights into pancreatic cancer-induced paraneoplastic diabetes. Nature Reviews Gastroenterology and Hepatology. 2013;10(7):423-33. [PubMed: 23528347]

2 Andersen DK, Korc M, Petersen GM, Eibl G, Li D, Rickels MR et al. Diabetes, Pancreatogenic Diabetes, and Pancreatic Cancer. Diabetes. 2017;66(5):1103-10. doi:10.2337/db16-1477. [PubMed: 28507210] This reports highlights key research gaps in relationship between PDAC and DM as identified by experts at a symposium held at the American Diabetic Association $76^{\text {th }}$ scientific session. 
3. Bosetti C, Rosato V, Buniato D, Zambon A, La Vecchia C, Corrao G. Cancer risk for patients using thiazolidinediones for type 2 diabetes: a meta-analysis. Oncologist. 2013;18(2):148-56. doi: 10.1634/theoncologist.2012-0302. [PubMed: 23345544]

4. Colmers IN, Bowker SL, Tjosvold LA, Johnson JA. Insulin use and cancer risk in patients with type 2 diabetes: a systematic review and meta-analysis of observational studies. Diabetes Metab. 2012;38(6):485-506. doi:10.1016/j.diabet.2012.08.011. [PubMed: 23159131]

5. Bodmer M, Becker C, Meier C, Jick SS, Meier CR. Use of antidiabetic agents and the risk of pancreatic cancer: a case-control analysis. Am J Gastroenterol. 2012;107(4):620-6. doi:10.1038/ajg. 2011.483. [PubMed: 22290402]

6. Singh S, Singh PP, Singh AG, Murad MH, McWilliams RR, Chari ST. Anti-diabetic medications and risk of pancreatic cancer in patients with diabetes mellitus: a systematic review and metaanalysis. Am J Gastroenterol. 2013;108(4):510-9; quiz 20. doi:10.1038/ajg.2013.7. [PubMed: 23399556]

7 Chen H, Zhou X, Chen T, Liu B, Jin W, Gu H et al. Incretin-Based Therapy and Risk of Pancreatic Cancer in Patients with Type 2 Diabetes Mellitus: A Meta-analysis of Randomized Controlled Trials. Diabetes Ther. 2016;7(4):725-42. doi:10.1007/s13300-016-0198-3. [PubMed: 27655330] The only meta-analysis including all randomized control trials of incretin-based therapy showing no short-term increased risk of PDAC in DM patients on incretin-based therapy.

8. Boniol M, Franchi M, Bota M, Leclercq A, Guillaume J, van Damme N et al. Incretin-Based Therapies and the Short-term Risk of Pancreatic Cancer: Results From Two Retrospective Cohort Studies. Diabetes Care. 2018;41(2):286-92. doi:10.2337/dc17-0280. [PubMed: 29146599]

9. Tseng CM, Liao WC, Chang CY, Lee CT, Tseng CH, Hsu YC et al. Incretin-based pharmacotherapy and risk of adverse pancreatic events in the ethnic Chinese with diabetes mellitus: A populationbased study in Taiwan. Pancreatology. 2017;17(1):76-82. doi:10.1016/j.pan.2016.10.003. [PubMed: 27743712]

10. Madiraju AK, Erion DM, Rahimi Y, Zhang XM, Braddock DT, Albright RA et al. Metformin suppresses gluconeogenesis by inhibiting mitochondrial glycerophosphate dehydrogenase. Nature. 2014;510(7506):542-6. doi:10.1038/nature13270. [PubMed: 24847880]

11. Chen K, Qian W, Jiang Z, Cheng L, Li J, Sun L et al. Metformin suppresses cancer initiation and progression in genetic mouse models of pancreatic cancer. Mol Cancer. 2017;16(1):131. doi: 10.1186/s12943-017-0701-0. [PubMed: 28738823]

12. Hu H, Fang Y, Zhou X, Gong L, Liu L, Wang W et al. Relationship of metformin with the risk of pancreatic cancer in patients with type 2 diabetes: a meta-analysis. Biomedical Research. 2017;28(10).

13. Wang Z, Lai ST, Xie L, Zhao JD, Ma NY, Zhu J et al. Metformin is associated with reduced risk of pancreatic cancer in patients with type 2 diabetes mellitus: a systematic review and meta-analysis. Diabetes Res Clin Pract. 2014;106(1):19-26. doi:10.1016/j.diabres.2014.04.007. [PubMed: 24837144]

14. Olson SH, Xu Y, Herzog K, Saldia A, DeFilippis EM, Li P et al. Weight Loss, Diabetes, Fatigue, and Depression Preceding Pancreatic Cancer. Pancreas. 2016;45(7):986-91. doi:10.1097/MPA. 0000000000000590. [PubMed: 26692445]

15. Azar M, Lyons TJ. Diabetes, insulin treatment, and cancer risk: what is the evidence? F1000 Med Rep. 2010;2. doi:10.3410/M2-4.

16. Mayer D, Shukla A, Enzmann H. Proliferative effects of insulin analogues on mammary epithelial cells. Arch Physiol Biochem. 2008;114(1):38-44. doi:10.1080/13813450801900645. [PubMed: 18465357]

17. Lee DY, Yu JH, Park S, Han K, Kim NH, Yoo HJ et al. The influence of diabetes and antidiabetic medications on the risk of pancreatic cancer: a nationwide population-based study in Korea. Sci Rep. 2018;8(1):9719. doi:10.1038/s41598-018-27965-2. [PubMed: 29946194]

18. Chan MT, Lim GE, Skovso S, Yang YH, Albrecht T, Alejandro EU et al. Effects of insulin on human pancreatic cancer progression modeled in vitro. BMC Cancer. 2014;14:814. doi: 10.1186/1471-2407-14-814. [PubMed: 25373319]

19. Horwitz RI, Feinstein AR. The problem of "protopathic bias" in case-control studies. Am J Med. 1980;68(2):255-8. [PubMed: 7355896] 
20. Drucker DJ. The biology of incretin hormones. Cell Metab. 2006;3(3):153-65. doi:10.1016/j.cmet. 2006.01.004. [PubMed: 16517403]

21. Inzucchi SE, Bergenstal RM, Buse JB, Diamant M, Ferrannini E, Nauck M et al. Management of hyperglycemia in type 2 diabetes: a patient-centered approach: position statement of the American Diabetes Association (ADA) and the European Association for the Study of Diabetes (EASD). Diabetes Care. 2012;35(6):1364-79. doi:10.2337/dc12-0413. [PubMed: 22517736]

22. Elashoff M, Matveyenko AV, Gier B, Elashoff R, Butler PC. Pancreatitis, pancreatic, and thyroid cancer with glucagon-like peptide-1-based therapies. Gastroenterology. 2011;141(1):150-6. doi: 10.1053/j.gastro.2011.02.018. [PubMed: 21334333]

23. Spranger J, Gundert-Remy U, Stammschulte T. GLP-1-based therapies: the dilemma of uncertainty. Gastroenterology. 2011;141(1):20-3. doi:10.1053/j.gastro.2011.05.019. [PubMed: 21723985]

24. Funch D, Gydesen H, Tornoe K, Major-Pedersen A, Chan KA. A prospective, claims-based assessment of the risk of pancreatitis and pancreatic cancer with liraglutide compared to other antidiabetic drugs. Diabetes Obes Metab. 2014;16(3):273-5. doi:10.1111/dom.12230. [PubMed: 24199745]

25. Gokhale M, Buse JB, Gray CL, Pate V, Marquis MA, Sturmer T. Dipeptidyl-peptidase-4 inhibitors and pancreatic cancer: a cohort study. Diabetes Obes Metab. 2014;16(12):1247-56. doi:10.1111/ dom.12379. [PubMed: 25109825]

26. Butler PC, Elashoff M, Elashoff R, Gale EA. A critical analysis of the clinical use of incretin-based therapies: Are the GLP-1 therapies safe? Diabetes Care. 2013;36(7):2118-25. doi:10.2337/ dc12-2713. [PubMed: 23645885]

27. Hart PA, Law RJ, Frank RD, Bamlet WR, Burch PA, Petersen GM et al. Impact of diabetes mellitus on clinical outcomes in patients undergoing surgical resection for pancreatic cancer: a retrospective, cohort study. Am J Gastroenterol. 2014;109(9):1484-92. doi:10.1038/ajg.2014.193. [PubMed: 25070053]

28 ๑. Sharma A, Smyrk TC, Levy MJ, Topazian MA, Chari ST. Fasting Blood Glucose Levels Provide Estimate of Duration and Progression of Pancreatic Cancer before Diagnosis. Gastroenterology. 2018. doi:10.1053/j.gastro.2018.04.025.This is the first study that estiamted the pre-diagnostic duration of invasive PDAC using blood glucose as a bio-marker of early PDAC and show hyperglycemia in PDAC preceeds by 30 to 36 months prior to PDAC diagnosis and this hyperglycemia is associated with a certain tumor volume.

29. Zhou PT, Li B, Liu FR, Zhang MC, Wang Q, Li YY et al. Metformin is associated with survival benefit in pancreatic cancer patients with diabetes: a systematic review and meta-analysis. Oncotarget. 2017;8(15):25242-50. doi:10.18632/oncotarget.15692. [PubMed: 28445955]

30. Zhou DC, Gong H, Tan CQ, Luo JQ. Prognostic significance of anti-diabetic medications in pancreatic cancer: A meta-analysis. Oncotarget. 2017;8(37):62349-57. doi:10.18632/oncotarget. 17728. [PubMed: 28977950]

31. Dong YW, Shi YQ, He LW, Cui XY, Su PZ. Effects of metformin on survival outcomes of pancreatic cancer: a meta-analysis. Oncotarget. 2017;8(33):55478-88. doi:10.18632/oncotarget. 18233. [PubMed: 28903435]

32. Xin W, Fang L, Fang Q, Zheng X, Huang P. Effects of metformin on survival outcomes of pancreatic cancer patients with diabetes: A meta-analysis. Mol Clin Oncol. 2018;8(3):483-8. doi: 10.3892/mco.2017.1541. [PubMed: 29468063]

33. Frouws MA, Sibinga Mulder BG, Bastiaannet E, Zanders MM, van Herk-Sukel MP, de Leede EM et al. No association between metformin use and survival in patients with pancreatic cancer: An observational cohort study. Medicine (Baltimore). 2017;96(10):e6229. doi:10.1097/MD. 0000000000006229. [PubMed: 28272215]

34. Jang WI, Kim MS, Kang SH, Jo AJ, Kim YJ, Tchoe HJ et al. Association between metformin use and mortality in patients with type 2 diabetes mellitus and localized resectable pancreatic cancer: a nationwide population-based study in korea. Oncotarget. 2017;8(6):9587-96. doi:10.18632/ oncotarget.14525. [PubMed: 28077783]

35 ๑. Chaiteerakij R, Petersen GM, Bamlet WR, Chaffee KG, Zhen DB, Burch PA et al. Metformin Use and Survival of Patients With Pancreatic Cancer: A Cautionary Lesson. J Clin Oncol. 2016;34(16):1898-904. doi:10.1200/JCO.2015.63.3511. [PubMed: 27069086] This study 
highlights the importance of patient selection and appropriate statistical analysis methods used when studying medication exposure and cancer survival.

36. Tan J, You Y, Guo F, Xu J, Dai H, Bie P. Association of elevated risk of pancreatic cancer in diabetic patients: A systematic review and meta-analysis. Oncol Lett. 2017;13(3):1247-55. doi: 10.3892/ol.2017.5586. [PubMed: 28454242]

37. Makhoul I, Yacoub A, Siegel E. Type 2 diabetes mellitus is associated with increased risk of pancreatic cancer: A veteran administration registry study. SAGE Open Med. 2016;4:2050312116682257. doi:10.1177/2050312116682257. [PubMed: 28348740]

38. Pannala R, Leirness JB, Bamlet WR, Basu A, Petersen GM, Chari ST. Prevalence and clinical profile of pancreatic cancer-associated diabetes mellitus. Gastroenterology. 2008;134(4):981-7. [PubMed: 18395079]

39. Chari ST, Leibson CL, Rabe KG, Ransom J, De Andrade M, Petersen GM. Probability of pancreatic cancer following diabetes: a population-based study. Gastroenterology. 2005;129(2): 504-11. [PubMed: 16083707]

40. Muniraj T, Chari ST. Diabetes and pancreatic cancer. Minerva Gastroenterol Dietol. 2012;58(4): 331-45. [PubMed: 23207610]

4100 . Aggarwal G, Rabe KG, Petersen GM, Chari ST. New-onset diabetes in pancreatic cancer: a study in the primary care setting. Pancreatology. 2012;12(2):156-61. doi:10.1016/j.pan. 2012.02.003. [PubMed: 22487526] This is the first study highlighting the importance of delays in diagnosis of diabetes by physcian's and its implications in early detection of PDAC.

42. Munigala S, Singh A, Gelrud A, Agarwal B. Predictors for Pancreatic Cancer Diagnosis Following New-Onset Diabetes Mellitus. Clin Transl Gastroenterol. 2015;6:e118. doi:10.1038/ctg.2015.44. [PubMed: 26492440]

43 0. Boursi B, Finkelman B, Giantonio BJ, Haynes K, Rustgi AK, Rhim AD et al. A clinical prediction model to assess risk for pancreatic cancer among patients with new-onset diabetes. Gastroenterology. 2017;152(4):840-50. e3. [PubMed: 27923728] This clinical risk prediction model uses physcian diagnosis of new-onset diabetes and based on various demographic, clinical and biochemical parameters at the time of NOD diagnosis predict 3-year risk of PDAC.

44. Pang Y, Kartsonaki C, Guo Y, Bragg F, Yang L, Bian Z et al. Diabetes, plasma glucose and incidence of pancreatic cancer: A prospective study of 0.5 million Chinese adults and a metaanalysis of 22 cohort studies. Int J Cancer. 2017;140(8):1781-8. doi:10.1002/ijc.30599. [PubMed: 28063165]

45 ๑. Sharma A, Kandlakunta H, Singh Nagpal SJ, Ziding F, Hoos W, Petersen GM et al. Model to Determine Risk of Pancreatic Cancer in Patients with New-onset Diabetes. Gastroenterology. 2018. doi:10.1053/j.gastro.2018.05.023.This is the first clinical risk prediction model that uses glycemic definition of new-onset DM and identifies a very high risk group for PDAC ( 4\% 3year risk) that may benefit from clinical work-up.

46. Harris MI, Klein R, Welborn TA, Knuiman MW. Onset of NIDDM occurs at least 4-7 yr before clinical diagnosis. Diabetes Care. 1992;15(7):815-9. [PubMed: 1516497]

47. Fraser LA, Twombly J, Zhu M, Long Q, Hanfelt JJ, Narayan KM et al. Delay in diagnosis of diabetes is not the patient's fault. Diabetes Care. 2010;33(1):e10. doi:10.2337/dc09-1129. [PubMed: 20040660]

48. Menke A, Casagrande S, Geiss L, Cowie CC. Prevalence of and Trends in Diabetes Among Adults in the United States, 1988-2012. JAMA. 2015;314(10):1021-9. doi:10.1001/jama.2015.10029. [PubMed: 26348752]

49. Chari ST. Detecting early pancreatic cancer: problems and prospects. Semin Oncol. 2007;34(4): 284-94. doi:10.1053/j.seminoncol.2007.05.005. [PubMed: 17674956]

50. CenterforDiseaseControl. https://gis.cdc.gov/grasp/diabetes/diabetesatlas.html accessed on January 17, 2018 https://gis.cdc.gov/grasp/diabetes/diabetesatlas.html.

51. Ortman JM, Velkoff VA, Hogan H. An aging nation: the older population in the United States.

52. Choe JW, Kim HJ, Kim JS, Cha J, Joo MK, Lee BJ et al. Usefulness of CA 19-9 for pancreatic cancer screening in patients with new-onset diabetes. Hepatobiliary Pancreat Dis Int. 2018;17(3): 263-8. doi:10.1016/j.hbpd.2018.04.001. [PubMed: 29752133] 
53. Murakami M, Nagai Y, Tenjin A, Tanaka Y. Proposed cut-off value of CA19-9 for detecting pancreatic cancer in patients with diabetes: a case-control study. Endocr J. 2018;65(6):639-43. doi:10.1507/endocrj.EJ17-0380. [PubMed: 29643268]

54. O'Brien DP, Sandanayake NS, Jenkinson C, Gentry-Maharaj A, Apostolidou S, Fourkala EO et al. Serum CA19-9 is significantly upregulated up to 2 years before diagnosis with pancreatic cancer: implications for early disease detection. Clin Cancer Res. 2015;21(3):622-31. doi: 10.1158/1078-0432.CCR-14-0365. [PubMed: 24938522]

55. Balasenthil S, Huang Y, Liu S, Marsh T, Chen J, Stass SA et al. A Plasma Biomarker Panel to Identify Surgically Resectable Early-Stage Pancreatic Cancer. J Natl Cancer Inst. 2017;109(8). doi:10.1093/jnci/djw341.

56. Pelaez-Luna M, Takahashi N, Fletcher JG, Chari ST. Resectability of presymptomatic pancreatic cancer and its relationship to onset of diabetes: a retrospective review of CT scans and fasting glucose values prior to diagnosis. Am J Gastroenterol. 2007;102(10):2157-63. doi:10.1111/j. 1572-0241.2007.01480.x. [PubMed: 17897335]

57. Gangi S, Fletcher JG, Nathan MA, Christensen JA, Harmsen WS, Crownhart BS et al. Time interval between abnormalities seen on $\mathrm{CT}$ and the clinical diagnosis of pancreatic cancer: retrospective review of CT scans obtained before diagnosis. AJR Am J Roentgenol. 2004;182(4): 897-903. doi:10.2214/ajr.182.4.1820897. [PubMed: 15039161]

58 . Bruenderman E, Martin RC, 2nd. A cost analysis of a pancreatic cancer screening protocol in high-risk populations. Am J Surg. 2015;210(3):409-16. doi:10.1016/j.amjsurg.2014.11.017. [PubMed: 26003200] This is the first study that evaluates the cost effectivness of screening with imaging in patients over the age of 50 years for PDAC.

59. National Lung Screening Trial Research T, Church TR, Black WC, Aberle DR, Berg CD, Clingan KL et al. Results of initial low-dose computed tomographic screening for lung cancer. N Engl J Med. 2013;368(21):1980-91. doi:10.1056/NEJMoa1209120. [PubMed: 23697514]

60. Kenner BJ, Chari ST, Maitra A, Srivastava S, Cleeter DF, Go VL et al. Early Detection of Pancreatic Cancer-a Defined Future Using Lessons From Other Cancers: A White Paper. Pancreas. 2016;45(8):1073-9. doi:10.1097/MPA.0000000000000701. [PubMed: 27518362]

61. Rex DK. Colonoscopy: a review of its yield for cancers and adenomas by indication. Am J Gastroenterol. 1995;90(3):353-65. [PubMed: 7872270] 


\section{Pancreatic cancer risk}

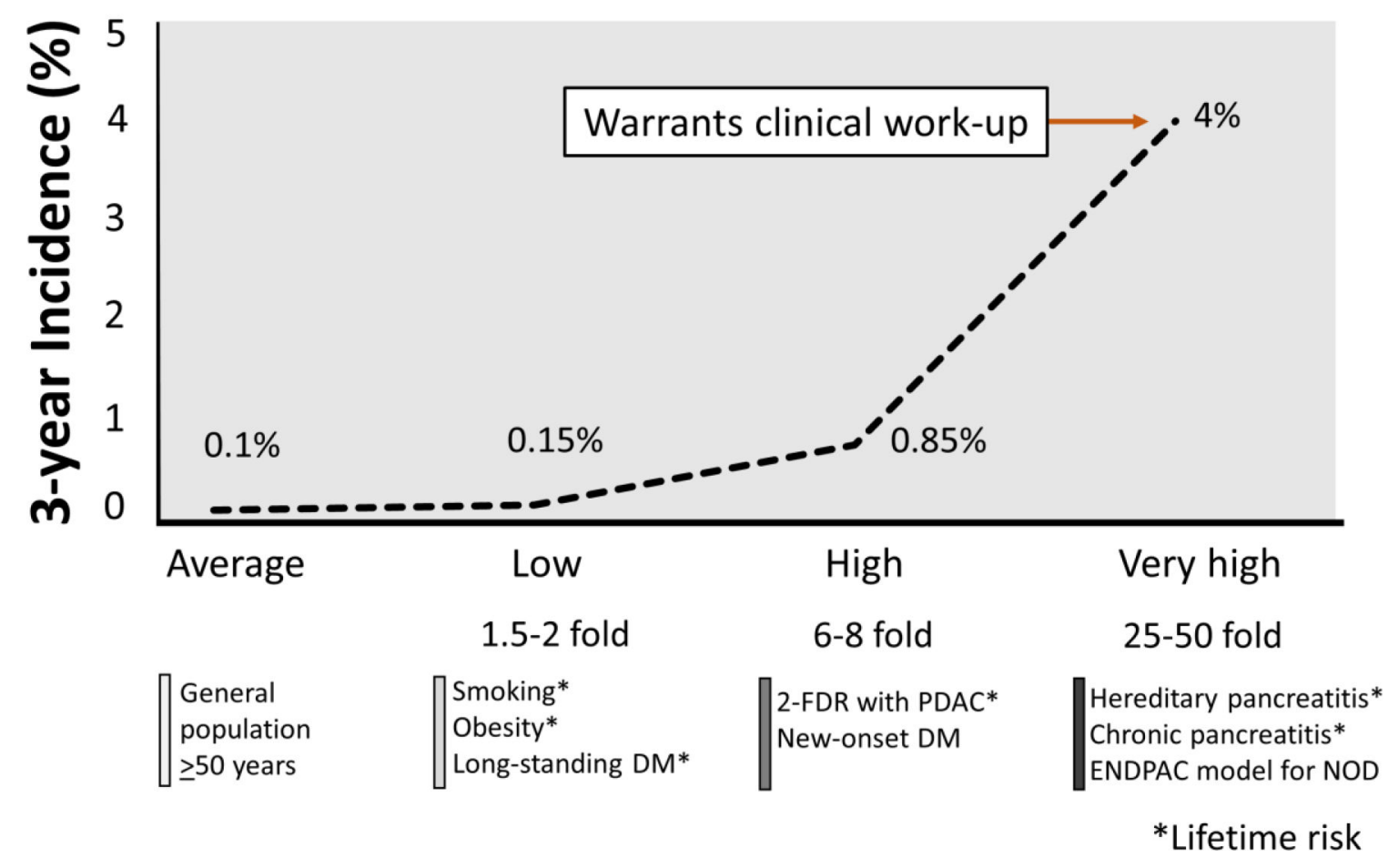

Figure 1.

Schematic diagram showing incidence risk of pancreatic cancer in currently known risk groups. Compared to long-standing diabetes, new-onset diabetes have a significantly higher risk. This risk further elevated with the clinical risk prediction model such as ENDPAC score. 